\title{
Suicidio consumado y representación de sus factores desencadenantes en la prensa generalista española en 2017
}

\section{Consummate suicide and representation of its triggering factors in the Spanish general press in 2017}

\section{María Teresa Santos}

Universidad del País Vasco, Leioa, España

mariateresa.santosaehu.eus

\section{Idoia Camacho}

Universidad del País Vasco, Leioa, España

idoia.camachodehu.eus

\section{Resumen}

Este artículo se centra en el análisis del tratamiento informativo que los diarios españoles El País, $A B C$, El Mundo y La Razón realizan sobre el suicidio consumado, concretamente sobre los factores desencadenantes del mismo, desde el 1 de enero de 2017 hasta el 31 de diciembre de 2017. Aplicando una metodología de análisis de contenido, los resultados muestran que los diarios analizados abordan el tema del suicidio consumado como la solución a un problema y, además, incluyen detalles como el mecanismo empleado y la nota de suicidio, llegando en algunos casos al sensacionalismo. Las informaciones reflejan los datos reales sobre suicidio, pero se alejan de las indicaciones de la OMS para tratar el tema desde una perspectiva más integral y menos noticiosa.

Palabras clave: Suicidio, España, periódicos, motivos suicidio, mecanismo empleado.

\begin{abstract}
This article focuses on the analysis of the informative treatment that the Spanish newspapers $E l$ País, ABC, El Mundo and La Razón gave to the consummate suicide, more precisely to the triggering factors, from the 1st of January to the 31st of December of 2017. Applying a methodology of content analysis, the results show that the analyzed journals pose the subject of suicide consummated as the solution to a problem and also include details such as the mechanism used and the suicide note. It could say that some cases are sensationalists. The information reflects the actual suicide data, but moves away from WHO's indications to treat the issue from a more comprehensive and less newsworthy perspective.
\end{abstract}

Keywords: Suicide, Spain, press, motives suicide, mechanism used. 


\section{Introducción}

El suicidio es la muerte producida por uno mismo con la intención precisa de poner fin a la propia vida (Villanueva \& Hernández, 2004, p. 708). La Organización Mundial de la Salud considera que el comportamiento suicida incluye el pensamiento de quitarse la vida, elaborar un plan, buscar los medios, intentar y llevar a cabo el suicidio (OMS, 2003, p. 2011. Conlleva una forma voluntaria en la que intervienen tanto los pensamientos suicidas lideación suicida) como el acto suicida en sí (Gutiérrez, Contreras \& Orozco, 2006, pp. 66-67), algo que no es fortuito y que tiene razones difíciles de descubrir (Ballús, 2006, pp. 4-5). Cada año más de 800 mil personas pierden la vida de este modo, resultando la segunda causa de muerte entre los jóvenes con edades comprendidas entre 15 a 29 años. En España, en 2017 se quitaron la vida unas 3.679 personas, lo que supone una tasa de 8,7 por cada 100.000 habitantes. En el mundo es de unos 12 y en Europa es del 11,4 (INE, 2017), de los cuales el 74,6 $\%$ son hombres (2.718) y el $25,4 \%$ mujeres (961). Es una cifra que duplica los accidentes de tráfico y que constituye la primera causa de muerte no natural en la población general con edad comprendida entre 15 y 34 años.

Se trata de un problema de salud pública en el que confluyen factores psicológicos, sociales, biológicos, culturales y ambientales (Hawton \& van Heeringen, 2009). No obstante, el suicidio ha constituido un tabú y la familia sufre la pérdida en silencio y los medios, en cierto modo durante décadas, han seguido esa política. Por eso, según González (2018), hoy es necesario que los medios de comunicación, asesorados por especialistas, sirvan a la sociedad y contribuyan a cambiar la percepción social que existe sobre el suicidio. La OMS elaboró una serie de recomendaciones destinadas a los medios de comunicación para la información sobre suicidios (2000). Entre otras indicaciones, destacan evitar el tratamiento sensacionalista y situar la noticia en lugar destacado, no usar la palabra "suicidio" en el titular, y ser prudentes en el uso de fotografías, vídeos o redes sociales y en publicar notas de suicidio por ninguna vía. Las recomendaciones de la OMS incluyen, también, no culpabilizar, no informar sobre detalles específicos como el método utilizado o el lugar del suicidio, así como no glorificar a la persona que se ha suicidado, ni presentarlo como algo normal ni como una solución a los problemas, ni como un modo de afron- tarlos, ni como una respuesta comprensible ante los cambios sociales o culturales.

La OMS indica la necesidad de incidir en su prevención, así como resaltar alternativas al suicidio, aportando información sobre cómo y dónde pedir ayuda, los recursos comunitarios y líneas de ayuda disponibles, así como también informar sobre factores de riesgo y señales de alarma. También contemplan la importancia de prestar atención a los sobrevivientes, difundir recursos donde puedan recibir apoyo, además de la conveniencia de transmitir la relación que existe entre la depresión y la conducta suicida, y que se puede tratar (Acosta, Rodríguez \& Cejas, 2017, p. 5). En cuanto al tratamiento del suicidio en los medios españoles el Código Deontológico de Asociaciones de Periodistas de España (FAPE), no hace ninguna referencia al suicidio. A su vez, los libros de Estilo de El País (2014, p. 33) y El Mundo (2002, p. 67), consideran que se debe publicar solo cuando se trata de personas de relevancia, mientras que $A B C$ no menciona el suicidio y La Razón carece de libro de estilo. Son numerosas las investigaciones desde el punto de vista de la psiquiatría que determinan que un alto porcentaje de suicidas padecía patologías psiquiátricas como trastornos de personalidad, ansiedad o depresión (Gelder, Gat \& Mayou, 1989; Roy, Nielse, Rylander, Sarchiapone \& Segal, 1999; Gándara, 1995; Morcillo, Saiz, Bousoño \& Bobes, 2000; Tejedor \& Sarró, 2005; Sadock \& Sadock, 2008; Mínguez, García \& Gándara, 2010, p. 146; y Gómez, Forti, Gutiérrez, Belmonte \& Martin, 2016). Otros aspectos que abordan las investigaciones son el comportamiento suicida (Nock et al. 2008), el método empleado para consumar el suicidio (Sudak \& Sudak, 2005; Pirkis, Blood, Beautrais, Burgess \& Skehams, 2006; Robledo, Koutsourais, López, Serrano, Villareal \& Martín, 2015), o las notas de suicidio (Chávez, Macías \& Luna, 2010; Casado, Mata \& Raya, 2012; y Acinas, Robles \& Peláez, 2015).

Centrándonos en la prensa, a raíz de la publicación de la novela de Goethe Die Leiden des jungen Werther (1774) se empieza a cuestionar la posibilidad de que los medios de comunicación puedan incrementar el número de suicidios. Relacionado con este tema en Estados Unidos, Motto (1967) tratan la sugestión del suicidio, mientras que Phillips (1974) analiza el llamado efecto Werther o de imitación. Considera que cuanto mayor es la publicidad que se le da, se incrementa el número de intentos posteriores (Phillips, Lesnya \& Paight, 1992; 
Müller 2011). En esta misma línea de aumento de suicidios consumados o intentos imitando los descritos en los medios inciden numerosos trabajos (Pirkis \& Blood, 2001; Dare, Andriessen, Nordentoft, Meier, Huisman \& Pirkis, 2011; Ladwig, Kunrath, Lukaschek \& Baumert, 2012; Sisask \& Värnik, 2012; y Scherr \& Steinleitner, 2015, entre otros muchos). Por el contrario, otros estudios indican que si se cubren las noticias de manera responsable puede resultar preventivo sobre la conducta suicida, lo que se conoce como el Efecto Papageno (Herrera, Ures \& Martínez, 2015, p. 125).

Respecto al tratamiento de las noticias sobre suicidio, un estudio sobre medios impresos en Colombia concluye que las informaciones publicadas no cumplen las recomendaciones de la OMS (Muñoz \& Sánchez, 2013; Rátiva, Ruiz \& Medina, 2013). En esta misma línea inciden los estudios de otros autores, aunque observan cambios positivos (Rubiano, Quintero \& Bonilla, 2007; Acosta, Rodríguez \& Cejas, 2017; Garrido, Eleazar \& Catalán, 2018). A su vez, Olmo y García (2015) establecen que la forma de tratar el suicidio en los medios puede hacer que se avance en la mejora de la opinión pública sobre el tema o, por el contrario, que no sirva para alcanzar la consecución de una utilidad común.

El suicidio tiene gran trascendencia social y son muy numerosas las investigaciones realizadas desde el área hospitalaria, forense y de la psiquiatría que abordan aspectos como los factores desencadenantes y el método empleado. Sin embargo, son más escasas las que se efectúan desde ámbito académico del área de la comunicación y más abordando estos aspectos concretos desde el punto de vista de su tratamiento informativo. De ahí la relevancia de este trabajo que lo analiza a través de la información publicada en los diarios de información general españoles El País, El Mundo, La Razón y $A B C$ durante el año 2017.

\section{Metodología}

\subsection{Objetivos}

El objetivo general de esta investigación es analizar el tratamiento informativo desplegado por la prensa española de información general sobre el suicidio consumado. Se han establecido los siguientes objetivos específicos: Cuantificar las pie- zas informativas publicadas sobre esta temática en los diarios objeto de análisis; comprobar si se informa sobre los factores que desencadenan el suicidio y averiguar cuáles son los factores predominantes; comprobar si se informa sobre el mecanismo empleado para el suicidio y averiguar cuál es el mecanismo predominante, y averiguar si los diarios respetan las recomendaciones de la OMS para informar sobre el suicidio.

\subsection{Metodología}

Para llevar a cabo el análisis se han elegido las cabeceras ${ }^{1}$ de El País (1976), El Mundo (1989), ABC (1903) y La Razón (1998). Se trata de periódicos de calidad, que integran grupos de comunicación consolidados, con cobertura nacional y líneas editoriales que cubren todos los espacios sociológicos. Por tanto, son periódicos de referencia y creadores de opinión. El espacio temporal analizado comprende desde el 1 de enero hasta el 31 de diciembre de 2017, fecha que la OMS presenta "Preventing suicide: a resource for media professionals - update" con recomendaciones para los medios de comunicación. Como unidad de análisis se contempla cada una de las piezas publicada en los diarios, con independencia del género periodístico empleado (informativo, interpretativo o de opinión), que contenga el término "suicido". La búsqueda de las noticias se ha realizado desde los portales web de los diarios mencionados. Tras la obtención de los primeros resultados, se han seleccionado todas las piezas que tenían como asunto principal el suicidio y se han descartado aquellas que tratan el tema del suicidio de manera circunstancial o utilizan el término como metáfora.

Después de la revisión hemerográfica, identificamos 878 piezas, de las cuales $347(39,5 \%)$ abordan el suicidio consumado, que son objeto de análisis: El País ( $\mathrm{n}=127,36,6 \%), A B C(\mathrm{n}=112,32,3 \%)$, El Mundo ( $n=62,17,9 \%$ ), y La Razón ( $n=46,13,2$ $\%)$. Fue preciso el diseño de una ficha específica, siguiendo trabajos previos (Miguel et al., 2005; Acinas, Robles \& Pelaéz, 2015; y Santurtun et al., 2016), con el fin de codificar los datos de acuerdo a las siguientes variables: fecha de publicación, procedencia, factores desencadenantes del suicidio Itrastornos depresivos, violencia de género, justicia/tribunales, corrupción, acoso, terrorismo, sin determinar); mecanismo empleado (arma de fue- 
go, ahorcadura, precipitación, sobreingesta medicamentosa, sofocación, explosión, sin determinar). También se contempló el tratamiento de la información: los titulares, cuerpo de la información y nota de suicidio. Con el fin de probar la fiabilidad del desarrollo de la codificación, se escogieron al azar 70 piezas (el $20 \%$ del total), de las que dos codificadores cifraron por separado cada una de las variables, obteniendo 67 y 66 coincidencias. El coeficiente Kappa de Cohen (K), arrojó una fiabilidad del $0,90 \%$ y un índice de confianza de $95 \%$. Son resultados que garantizan la fiabilidad ( $\geqslant 80$ ), según autores como Landis \& Koch (1977, e Igartua (2006, p. 221). Se utilizó como método el análisis de contenido por considerar su aplicación adecuada a este tipo de investigaciones. Una técnica propuesta por autores como: Berelson (1952), Holsti (1969), Krippendorf (1980), Bardin (1986), Piñuel \& Gaitán (1995) e Igartua (2006). Además, posibilita el estudio de los textos publicados de una forma sistemática, objetiva y cuantitativa (Wimmer \& Dominick, 1996 , p. 170), y permite obtener datos relevantes (Bardin \& Suárez, 2002, p. 7; Gonzáles, 2015, pp. 321-328; y Piñuel, 2002, pp. 1- 42).

\section{Resultados}

\subsection{El suicidio consumado en la agenda de los medios}

Durante el año 2017 se han contabilizado 347 textos correspondientes al suicidio consumado $(E l$ País 36,6\%, ABC 32,3 \%, El Mundo 17,9\% y La Razón $13,2 \%$ ), de los cuales el $27 \%$ se refiere a personajes famosos (El País 31\%, ABC 31\%, El Mundo 23\% y La Razón 10\%).
En el conjunto de periódicos, se publica un mayor número de noticias en julio $(20,2 \%)$ y diciembre $(11,3 \%)$. Todos los diarios coinciden en publicar más noticias el mes de julio (La Razón 21,8 \%, El Mundo 22,7 \%, ABC 21,4\% y El País 17,35\%). Por el contrario, el mes de junio es el que menos atención mediática centra (El País 7,9%, La Razón 2,2 $\%$, El Mundo 1,6 \% y ABC 0,9\%). Al analizar la procedencia de la información destaca el ámbito estatal $(53,89 \%)$, aunque con diferencias entre los diarios (La Razón 71,7 \%, El Mundo 58 \%, ABC 54,4 \% y El País 45,6\%). Por comunidades, tiene mayor cobertura Madrid (18,4\%), Andalucía $(9,2 \%$ ) y Región de Murcia (4,3\%). Las informaciones procedentes del ámbito internacional $(45,8 \%)$, encabezadas por Estados Unidos (20,4\%), seguido de los países latinoamericanos (4,6\%), Reino Unido (3,4\%), Corea (2,3\%), Holanda, Italia y Rusia ( $2 \%$ cada una) y Japón $(1,5 \%)$.

\subsection{Factores desencadenantes del suicidio}

En cuanto al sexo, en las informaciones analizadas el $46,1 \%$ de las personas que se suicidan son hombres y el $31,4 \%$ mujeres, con edades comprendidas entre 20 y 40 años (23\%). Los casados suponen el $20,2 \%$ del total. Entre los factores desencadenantes del suicidio, tal y como se refleja en la Tabla 1, ocupan el primer lugar los trastornos mentales/ depresivos, en el 18,44\% del total de noticias, seguidos de los problemas relacionados con la violencia de género con el $12,10 \%$ y la corrupción con $8 \%$.

Tabla 1. Factores desencadenantes del suicidio

\begin{tabular}{|l|c|c|c|c|c|c|c|c|c|c|}
\hline & \multicolumn{2}{|c|}{ La Razón } & \multicolumn{2}{c|}{ El Mundo } & \multicolumn{2}{c|}{ El País } & \multicolumn{3}{c|}{$A B C$} & \multicolumn{2}{c|}{ Total } \\
\cline { 2 - 14 } & $\begin{array}{l}\text { Núm. } \\
\text { piezas }\end{array}$ & $\%$ & $\begin{array}{l}\text { Núm. } \\
\text { piezas }\end{array}$ & $\%$ & $\begin{array}{l}\text { Núm. } \\
\text { piezas }\end{array}$ & $\%$ & $\begin{array}{l}\text { Núm. } \\
\text { piezas }\end{array}$ & $\%$ & $\begin{array}{l}\text { Núm. } \\
\text { piezas }\end{array}$ & $\%$ \\
\hline T. depresivos & 3 & 6,52 & 20 & 32,2 & 24 & 18,89 & 17 & 15,17 & 64 & 18,44 \\
\hline Violencia de género & 2 & 4,34 & 2 & 3,22 & 24 & 18,89 & 14 & 12,5 & 42 & 12,10 \\
\hline Justicia/Tribunales & 2 & 4,34 & 8 & 12,90 & 12 & 9,44 & 16 & 14,28 & 38 & 10,95 \\
\hline Corrupción & 6 & 13,04 & 3 & 4,83 & 8 & 6,29 & 11 & 9,82 & 28 & 8,06 \\
\hline Acoso & 6 & 13,04 & 5 & 8,06 & 8 & 6,29 & 8 & 7,14 & 27 & 7,90 \\
\hline Terrorismo & 2 & 4,34 & 3 & 4,83 & 1 & 0,78 & 2 & 1,78 & 8 & 2,30 \\
\hline Sin determinar & 25 & 54,34 & 21 & 33,87 & 50 & 39,37 & 44 & 39,28 & 140 & 40,34 \\
\hline TOTAL & 46 & 100 & 62 & 100 & 127 & 100 & 112 & 100 & 347 & 100 \\
\hline
\end{tabular}

Fuente: Elaboración propia 


\subsubsection{Trastornos depresivos}

Según estimaciones de la Organización Mundial de la Salud, unos 300 millones de personas padecen depresión (WHO, 2017) una enfermedad que puede afectar el trabajo y la vida personal e incrementar el riesgo de padecer otras dolencias e incluso en los casos más graves el suicidio. Estudios sobre la autopsia psicológica constatan que entre el 90 y $95 \%$ de los casos de suicidio existe al menos un diagnóstico psiquiátrico al momento del suicidio (Nock et al., 2008; y Forti- Sampiero \& FortiBuratti, 2012). Como se observa en la Tabla 1, los problemas originados por los trastornos depresivos mentales $(18,4 \%)$ se decantan como las causas más frecuentes, porcentaje que se incrementa hasta el 40,5\% en las noticias sobre personajes famosos (El Mundo 54,6\%, El País 45,2\%, ABC 32,2\% y La Razón 20\%). Corresponde a un sector de población con edad comprendida entre 20 y 29 años y hay un mayor porcentaje de hombres $(60,9 \%)$ que de mujeres $(28,2 \%)$, aunque con diferencias entre los diarios. Predominan los hombres en (La Razón $100 \%$, El País $87,5 \%$ y ABC $52,9 \%$ ) y en El Mundo hombres y mujeres alcanzan el mismo porcentaje ( $45 \%$ cada uno) y en el $10,9 \%$ de las notas, no se determina el sexo.

\subsubsection{Violencia de género}

Consideramos como tal "todo acto de violencia sexista que tiene como resultado posible o real un daño físico, sexual o psíquico, incluidas las amenazas, la coerción o la privación arbitraria de libertad, ya sea que ocurra en la vida pública o en la privada" (ONU, 1995). En los diarios analizados, esta causa supone el 12,1\% del total (El País 18,9\%, ABC $12,5 \%$, La Razón 4,3\% y El Mundo 3,2\%), con una mayoría de hombres (78,6\%), (El País $83,3 \%$, La Razón $81,8 \%$ y $A B C$ 81,2\%), con una edad comprendida entre 40 y 49 años $(28,6 \%)$ y entre 20 y 39 años (42,8\%). A su vez, en las mujeres alcanza el 11,9\% (El Mundo 50\%, ABC 18,8\%, La Razón 18,2\% y El País $4,1 \%$ ) y en el $28,5 \%$ de los casos su edad está comprendida 40 y 49 años y el $42,8 \%$ entre 20 y 39 años. Cabe destacar que entre los agresores por violencia de género se detecta una elevada tasa de suicidios $(21,15 \%)$, según la Memoria del Fiscal General del Estado.

\subsubsection{Justicia/Tribunales}

En esta temática se enmarcan las noticias referentes a sentencias y procesos judiciales relacionados con el tema. Supone el 10,9\% del total (ABC 14,2 \%, El Mundo 12,9 \%, El País 9,4 \% y La Razón 4,3 $\%)$, cifras que descienden cuando los protagonistas son famosos (4,2\%). Hay una mayoría de hombres $(57,9 \%)$, con una edad que supera los 70 años (ABC 68,7\%, El Mundo 62,5\%, La Razón (50\%), y El País $41,7 \%$ ). El porcentaje de mujeres resulta poco significativo $(5,3 \%)$ y solo aparecen en El Mundo $(12,5 \%)$ y $A B C(6,3 \%)$.

\subsubsection{Corrupción}

Se abordan noticias relativas al abuso de poder público para obtener beneficios privados, a través de decisiones favorables por parte del poder (Código Penal LO 1/2015). Constituyen un $8,06 \%$ de los textos (La Razón 13\%, ABC 9,8\%, El País 6,2\% y El Mundo 4,8\%). En cuanto a los hombres supone el $85,1 \%$, de los cuales el $39,2 \%$ tienen una edad comprendida entre 60 y 69 años, alcanzando porcentajes elevados en todos los diarios (La Razón $100 \%$, El País 100\%, El Mundo $75 \%$ y ABC $72,7 \%$ ). No se publica ningún caso de corrupción en mujeres y en el $27,3 \%$ no se determina.

\subsection{5. $A \cos 0$}

Entendemos como acoso las conductas de gravedad que se pueden dar de manera reiterada como coacciones o amenazas, que menoscaba la libertad, la dignidad, implica persecuciones, seguimientos o vigilancias, hostigamiento que puedan alterar la vida cotidiana, reconocido como delito por el Código Penal (LO 1/2015, de 30 de marzo). Estas noticias alcanzan el 7,9\% del total (La Razón 13\%, El Mundo 8\%, ABC 7,1\% y El País 6,2\%). La edad oscila entre 8 y 17 años $(55,5 \%)$ y esta causa es más frecuente en mujeres $(66,6 \%)$, como se refleja en El Mundo (80\%), ABC (75\%), El País (50\%) y La Razón $(66,6 \%)$. En el caso de los hombres es inferior $25 \%$. 


\subsubsection{Terrorismo}

Esta temática alcanza porcentajes poco significativos en la muestra (El Mundo 4,8\%, La Razón $4,3 \%$ y $A B C 1,7 \%$ y El País $0,8 \%$ ). No registra ningún caso de mujeres y en la mitad de los artículos analizados, no se especifica el sexo.

\subsection{Mecanismo empleado para consumar el suicidio}

La OMS recomienda no ahondar en los métodos empleados ni en el lugar en los contenidos informativos sobre suicidio para evitar que personas vulnerables puedan copiar el modus operandi (OMS, 2017). Tal y como se refleja en la Tabla 2 , en el $62,5 \%$ de los artículos analizados se especifica el mecanismo de los suicidios larmas de fuego $20,1 \%$, la ahorcadura $16,4 \%$ y la precipitación $11,5 \%$ ). Sin embargo, se constatan discrepancias entre periódicos: en La Razón predomina la precipitación (17,3\%), en El Mundo el ahorcamiento $(20,9 \%)$, mientras que $A B C$ y El País coinciden en las armas de fuego (22\% y $22,3 \%$ cada uno). Cabe destacar que la primera opción para los famosos es la ahorcadura $(26,6 \%)$, seguida del arma de fuego $(11,8 \%)$ y la sofocación $(7,4 \%)$. Otros métodos como la precipitación, explosión o sobreingesta medicamentosa suponen porcentajes inferiores $(2,1 \%)$. En cuanto al sexo, los hombres emplean más las armas de fuego $(87,2 \%)$ y el ahorcamiento $(61,4 \%)$, mientras que las mujeres optan por el ahorcamiento $(29,8 \%)$ y los fármacos $(26,1 \%)$ en las notas publicadas.

\subsubsection{Armas de fuego}

Es la mención predominante, con un $20,1 \%$ de los casos (El País 22\%, ABC 22,3\%, El Mundo 19,3\% y La Razón 10,8\%), más empleado por los hombres $(87,2 \%)$ que por las mujeres $(5,7 \%)$. En los suicidios que emplean este mecanismo se destacan como motivos los problemas relacionados con la corrupción $(27,1 \%)$ y la justicia/ tribunales $(17,2 \%)$, aunque con discrepancias entre los diarios. Así en La Razón y $A B C$ predomina la corrupción $180 \%$ y $28 \%$, respectivamente), en El Mundo los trastornos depresivos (25\%) y en El País los temas relacionados con la justicia/tribunales $(21,4 \%)$. En cuanto a la edad, destacan los mayores de 60 años $(34,2 \%)$, datos que coinciden en todos los diarios (La Razón 60\%, ABC 36\%, El Mundo 33,3\% y El País 28,6\%).

\subsubsection{Ahorcadura}

La asfixia por ahorcadura se especifica en $16,4 \%$ del total de piezas (El Mundo 20,9\%, El País 19,6\%, ABC $12,5 \%$ y La Razón $10,8 \%$ ). Como motivos que pueden desencadenar el suicidio destacan los trastornos depresivos $(24,6 \%)$ y la violencia de género $(19,3 \%)$. Son más los hombres $(61,4 \%)$ que las mujeres $(29,8 \%)$ que ponen fin a su vida de este modo y su edad está comprendida entre los 30 y 49 años $(42 \%)$.

Tabla 2. Mecanismo empleado para consumar el suicidio

\begin{tabular}{|l|c|c|c|c|c|c|c|c|c|c|}
\hline & \multicolumn{2}{|c|}{ La Razón } & \multicolumn{2}{c|}{ El Mundo } & \multicolumn{2}{c|}{ El País } & \multicolumn{2}{c|}{$A B C$} & \multicolumn{3}{c|}{ Total } \\
\cline { 2 - 13 } & $\begin{array}{l}\text { Núm. } \\
\text { piezas }\end{array}$ & $\%$ & $\begin{array}{l}\text { Núm. } \\
\text { piezas }\end{array}$ & $\%$ & $\begin{array}{l}\text { Núm. } \\
\text { piezas }\end{array}$ & $\%$ & $\begin{array}{l}\text { Núm. } \\
\text { piezas }\end{array}$ & $\%$ & $\begin{array}{l}\text { Núm. } \\
\text { piezas }\end{array}$ & $\%$ \\
\hline Arma de fuego & 5 & 10,86 & 12 & 19,35 & 28 & 22,04 & 25 & 22,32 & 70 & 20,17 \\
\hline Ahorcadura & 5 & 10,86 & 13 & 20,96 & 25 & 19,68 & 14 & 12,50 & 57 & 16,42 \\
\hline Precipitación & 8 & 17,39 & 8 & 12,90 & 4 & 3,14 & 20 & 17,85 & 40 & 11,52 \\
\hline Sobreingesta medicamentos & 4 & 8,69 & 9 & 14,51 & 8 & 6,29 & 2 & 1,78 & 23 & 6,62 \\
\hline Sofocación & 3 & 6,52 & 5 & 8,06 & 5 & 3,93 & 7 & 6,25 & 20 & 5,76 \\
\hline Explosión & 0 & 0 & 4 & 6,45 & 1 & 0,78 & 2 & 1,78 & 7 & 2,01 \\
\hline Sin determinar & 21 & 45,65 & 11 & 17,74 & 56 & 44,09 & 42 & 37,50 & 130 & 37,46 \\
\hline TOTAL & 46 & 100 & 62 & 100 & 127 & 100 & 112 & 100 & 347 & 100 \\
\hline
\end{tabular}

Fuente: Elaboración propia 


\subsubsection{Precipitación}

Se entiende como tal arrojarse desde un lugar alto. Supone el $11,5 \%$ de las piezas publicadas, aunque con proporciones muy diferentes entre los diarios IABC 17,8 \%, La Razón 17,4\%, El Mundo 12,9\% y El País 3,1\%). Los motivos principales son la violencia de género $(22,5 \%)$, que supone mayor porcentaje en hombres $(57,5 \%)$ que en mujeres (25\%). Su edad oscila entre 20 y 39 años (32,5\%). En La Razón se trata sobre todo de menores de 29 años (62,5\%), en $A B C$ son menores de 40 años (45\%) y en El País la edad varía entre 30 y 49 años (75\%).

\subsubsection{Sobreingesta medicamentosa}

En este apartado se incluyen noticias que utilizan como mecanismo de suicidio la ingesta de fármacos o tóxicos. Suponen el $6,6 \%$, aunque con diferencias entre los diarios (El Mundo 14,5\%, La Razón 8,6\%, El País 6,9\% y $A B C$ 1,78\%). Es empleado por sujetos con edades comprendidas entre $40 \mathrm{y}$ 59 años (26\%) y mayores de 70 (21,7\%), aunque de nuevo los diarios marcan diferencias. Así, en $A B C$ (50\%) y La Razón (25\%) predomina la edad comprendida entre 40 y 49 años, El Mundo sobrepasa los $70(44,5 \%)$ y en El País oscila entre 50 y 59 $(37,5 \%)$. Entre los motivos se alude a trastornos depresivos $(26,1 \%)$ seguido de violencia de género y justicia/tribunales ( $13,5 \%$ cada uno).

\subsubsection{Sofocación}

Se entiende como tal la obstrucción facial, compresión de tórax o la terminación de oxígeno en un contenedor cerrado (Celis, Hernández, Gómez,
Orozco \& Rivas, 2004: 503). Alcanza el 5,7\% de las piezas (El Mundo 8\%, La Razón 6,5\%, ABC 6,2\% y El País 3,9\%). En cuanto a los motivos, destacan los trastornos depresivos (20\%), resultado en el que coinciden todos los diarios (El País 100\%, La Razón $45 \%, A B C 42,8 \%$ y El Mundo 20\%), seguido de justicia/tribunales (20\%). Se trata de hombres $(60 \%)$ y mujeres $(10 \%)$ con edad entre 20 y 29 años $(18,5 \%)$, aunque se muestran diferencias entre los diarios. Así en El Mundo predomina una edad entre 18 y 19 años $(11,1 \%)$ y más de 60 (11,1\%), en El País entre 20 y 29 años (50\%), en $A B C$ menos de $29(22,2 \%)$ y en La Razón (18,5\%).

\section{Otras recomendaciones de la OMS}

La OMS recomienda evitar el uso de la palabra suicidio en el titular. Sin embargo, en los diarios analizados en el $29,3 \%$ de los casos se menciona el término suicidio ( $E$ l Mundo $38,7 \%, A B C 32,2 \%$, El País 24,4\% y La Razón 23,9\%), porcentaje que desciende al $21,3 \%$ cuando son personajes famosos (ABC 29\%, El Mundo 22,7\%, El País 16,2\% y La Razón $10 \%$ ). A su vez, no se incluye en el $35,8 \%$, aunque con diferencias significativas entre los diarios (La Razón 45,7\%, El País 44,1\%, El Mundo 29\% y $A B C 25,9 \%$ ). En cuanto a los titulares, en el $34,8 \%$ de la muestra analizada se emplean acepciones como muerto (El País 77,5\%, ABC 68,8\%, El Mundo $65,5 \%$ y La Razón $64,2 \%)$, se quita la vida $(14,8 \%)$, ahorcado $(8,51 \%)$ cuerpo y fallecido $(4,25 \%$ cada uno). En el caso de los famosos, en el $55,3 \%$ no se especifica en el titular y en el $23,4 \%$ sí (ABC 29\%, El Mundo 22,7\% El País 16,4\% y La Razón 10\%).

En cuanto al cuerpo de la información, en el 62,6\% de los casos se determina el mecanismo empleado para consumar el suicidio y además en el $52,7 \%$ se

Tabla 3. Mención del término suicidio en los titulares

\begin{tabular}{|l|c|c|c|c|c|c|c|c|c|c|}
\hline & \multicolumn{2}{|c|}{ La Razón } & \multicolumn{2}{c|}{ El Mundo } & \multicolumn{2}{c|}{ El País } & \multicolumn{3}{c|}{$A B C$} & \multicolumn{2}{c|}{ Total } \\
\cline { 2 - 13 } & $\begin{array}{l}\text { Núm. } \\
\text { piezas }\end{array}$ & $\%$ & $\begin{array}{l}\text { Núm. } \\
\text { piezas }\end{array}$ & $\%$ & $\begin{array}{l}\text { Núm. } \\
\text { piezas }\end{array}$ & $\%$ & $\begin{array}{l}\text { Núm. } \\
\text { piezas }\end{array}$ & $\%$ & $\begin{array}{l}\text { Núm. } \\
\text { piezas }\end{array}$ & $\%$ \\
\hline Se menciona & 21 & 45,7 & 18 & 29,1 & 56 & 44,1 & 29 & 25,8 & 124 & 35,8 \\
\hline No se menciona & 11 & 23,91 & 24 & 38,70 & 31 & 24,40 & 36 & 32,14 & 102 & 29,39 \\
\hline Otras acepciones & 14 & 30,43 & 20 & 32,25 & 40 & 31,49 & 47 & 41,96 & 121 & 34,87 \\
\hline TOTAL & 46 & 100 & 62 & 100 & 127 & 100 & 112 & 100 & 347 & 100 \\
\hline
\end{tabular}

Fuente: Elaboración propia 
ofrecen detalles sobre el mismo (El Mundo 62,9\%, ABC 58\%, El País 48\% y La Razón 39,1\%).

La OMS también recomienda no tratar el suicidio de forma sensacionalista, así como evitar aportar información pormenorizada sobre el lugar del suicidio. No obstante, en el $9,7 \%$ de las piezas se presentan detalles que pueden considerarse sensacionalistas (El Mundo 19,3\%, La Razón 15,2\%, El País $7,8 \%$ y $A B C 4,4 \%$ ), es decir se ahonda en detalles escabrosos con el fin de intentar producir emociones intensas en el lector (Acinas, Robles \& Peláez, 2015: 74).

Algunos ejemplos son:

Un hombre de 47 años ha sido hallado muerto en Toledo dentro de un vehículo este mediodía. El fallecido tenía una bolsa de plástico tapando su cabeza y una bombona con gas conectada a un tubo de plástico, según confirmó a abc.es la Policía Nacional. Se trataba de dióxido de carbono, apuntaron otras fuentes (ABC, 22/11/2017).

"Una mujer y sus hijos aparecen estrangulados en su casa y el marido muere tras arrojarse por la ventana". "Al asomarse por el balcón vio a un hombre en el suelo. Se trataba de su vecino: Manuel Bustamante, que se había precipitado en ropa interior -en calzoncillos y en calcetines- desde un segundo piso (La Razón, 03/2017).

"Los cabos sueltos del misterioso "caso Lesvy"." "Sobre la fría mesa del forense yace el cuerpo desnudo de Lesvy Berlín Osorio. Sus ojos están cerrados suavemente y su boca, entreabierta por unos labios resecos. Su pómulo izquierdo tensa una herida que se ha vuelto negra, con escamas, y el lado izquierdo de su cuello ha quedado grabado para siempre por el profundo y delgado surco de un cable de teléfono" (El País, 13/07/2017).

Otra recomendación de la OMS se centra en evitar la publicación de notas suicidas en cualquiera de sus posibles modalidades. En los textos analizados la nota de despedida aparece en el 5,5\% (El Mundo 6,5\%, El País 6,3\%, ABC 4,5\%, y La Razón 4,4\%). Sin embargo, el porcentaje se incrementa hasta el 14,9 $\%$ cuando se trata de famosos (El Mundo 27,3\%, El País $16,2 \%$ y $A B C 9,7 \%)$.

Algunos ejemplos son:

Kim Jong-hyun, el cantante de la banda Shinee, dejó una carta de despedida antes de morir que decía: “Estoy roto por dentro. La depresión que lentamente me ha ido carcomiendo ya me ha devorado, y no he podido superarla. Es increíble lo mucho que duele. Nadie está más atormentado ni debilitado que yo" (El País, 19/12/2017).

Tan acosador es el joven que graba y se ríe como el que golpea. Era, para sus compañeros, «gorda y fea» y ella se sentía terriblemente desgraciada, reza su propia nota de suicidio (ABC 3/2/2017).

\section{Discusión y conclusiones}

Este artículo tuvo como objetivo el análisis del tratamiento informativo que realizan los diarios generalistas españoles sobre el suicidio consumado, centrado en los factores desencadenantes y el me-

Tabla 4. Alusiones a la nota de suicidio

\begin{tabular}{|l|c|c|c|c|c|c|c|c|c|c|}
\hline & \multicolumn{2}{|c|}{ La Razón } & \multicolumn{2}{c|}{ El Mundo } & \multicolumn{2}{c|}{ El País } & \multicolumn{2}{c|}{$A B C$} & \multicolumn{2}{c|}{ Total } \\
\cline { 2 - 11 } & $\begin{array}{l}\text { Núm. } \\
\text { piezas }\end{array}$ & $\%$ & $\begin{array}{l}\text { Núm. } \\
\text { piezas }\end{array}$ & $\%$ & $\begin{array}{l}\text { Núm. } \\
\text { piezas }\end{array}$ & $\%$ & $\begin{array}{l}\text { Núm. } \\
\text { piezas }\end{array}$ & $\%$ & $\begin{array}{l}\text { Núm. } \\
\text { piezas }\end{array}$ & $\%$ \\
\hline Sin nota & 44 & 95,65 & 58 & 93,54 & 119 & 93,70 & 107 & 95,53 & 328 & 94,52 \\
\hline Con nota & 2 & 4,35 & 4 & 6,46 & 8 & 6,30 & 5 & 4,47 & 19 & 5,48 \\
\hline TOTAL & 46 & 100 & 62 & 100 & 127 & 100 & 112 & 100 & 347 & 100 \\
\hline
\end{tabular}

Fuente: Elaboración propia 
canismo empleado. Es relevante conocer la cobertura que tiene el suicidio en la agenda de los medios de comunicación y saber si, además, tiene una finalidad social como propone McCombs (2006).

El estudio demuestra que el suicidio es un tema de interés en la prensa estatal, dado que se publican noticias todos los meses, aunque acaparan un mayor número de noticias en julio y diciembre, fechas coincidentes con el suicidio de famosos ( $\mathrm{He}$ rrera, Ures \& Martínez, 2015). Sin embargo, cabe destacar que el número de suicidios consumados solo en España durante 2017 es de 3.679, de modo que la mayor parte de informaciones se refieren a casos relacionados con personajes famosos o aquellos que tienen características noticiosas o de interés general. Unos resultados que coinciden con las recomendaciones de los libros de Estilo de El País (2014, p. 33) y El Mundo (2002, p.67), mientras que $A B C$ no menciona el suicidio y La Razón no tiene libro de estilo. A su vez el Código Deontológico Europeo de la Profesión Periodística y la FAPE no hacen referencia de manera explícita sobre el suicidio. Numerosos estudios concluyen que se ha producido un incremento de piezas publicadas sobre suicidio consumado (Garrido, Eleazar \& Catalán, 2018, p.815; y Herrera, Ures \& Martínez, 2015, p.130). Un incremento que sugiere que el suicidio deja de ser tabú, tras décadas de silencio en los medios y con ello sirven a la sociedad (González, 2018).

El suicidio se presenta en la mayoría de los casos como una respuesta ante un problema. Son numerosos los estudios que constatan que los trastornos psicológicos de personalidad, ansiedad y depresión constituyen la primera causa de muerte IGelder, Gat \& Mayou, 1989; Roy, Nielse, Rylander, Sarchiapone \& Segal, 1999; Sadock \& Sadock, 2008; Mínguez, García \& Gándara, 2010; Gómez, Forti, Gutiérrez, Belmonte \& Martin, 2016). También coinciden los trabajos realizados con datos forenses o psicológicos (Dorpat \& Ripley, 1960; Barraclough, Bunh, Nelson \& Sainsbury, 1974; Arató, Demeter, Rihmer \& Somogy, 1988; Rich, Ricketts, Fowler \& Young, 1988). Los resultados de la investigación confirman la evidencia de que la depresión es el trastorno mental individual que con mayor frecuencia precede al acto suicida y por ello conlleva más riesgo (Casado, Mata \& Raya, 2012, p.3). Unos datos que coinciden con Garrido et. al (2018, p. 815), pero son superiores a los obtenidos por Muñoz \& Sánchez (2013, p.16). Estos resulta- dos muestran que los diarios analizados informan sobre los motivos que impulsan a consumar el suicidio y con ello están alejados de las recomendaciones de la OMS en su programa SUPRE (SUICIDE PREVENTION), para la prevención del suicidio ( $\mathrm{Na}-$ varro, 2017, p. 29).

En cuanto al modus operandi empleado, este se especifica en el $62,5 \%$ de los casos, datos inferiores a los obtenidos por Muñoz \& Sánchez (2013, p.16) $(74,8 \%)$. Destacan los métodos violentos como armas de fuego, precipitación, ahorcadura, frente a otros no violentos como sobreingesta de fármacos u otras sustancias y sofocación o gases (Gómez et. al., 2016; Herrera, Ures \& Martínez, 2015). Otros estudios demuestran que predominan los métodos violentos (Miguel et al. 2005, p. 6; Gómez, Forti, Gutiérrez, Belmonte \& Martin, 2014; Santurtún, Santurtún, Agudo \& Zarrabeitia, 2016; e INE, 2017). Estos datos no coinciden exactamente con los resultados del estudio realizado en el año 2000 por Sánchez, Orejarena \& Guzmán (2004, p.220) y de las investigaciones de Miguel et al. (2005, p.46) y de Romero \& Gamero (2005, p.7), según los cuales ocupa el primer lugar la ahorcadura, seguido de las armas de fuego. Por tanto, es una realidad que los diarios analizados publican información sobre el mecanismo empleado para consumar el suicidio, algo que resulta contrario a las recomendaciones dadas por la OMS, con el fin de evitar favorecer conductas suicidas (Sudak \& Sudak, 2005; Pirkis, Blood, Beautrais, Burgess \& Skehams, 2006). De nuevo los medios no cumplen las recomendaciones de la OMS en cuanto a que informan sobre detalles y descripciones del mecanismo empleado.

En los diarios analizados se menciona el término suicidio en el titular $(29,3 \%)$ junto con otras acepciones como muerto, quitarse la vida, así como alusiones al mecanismo empleado para consumar el suicidio, que en el cuerpo de la noticia alcanzan el $52,7 \%$, junto con detalles que se pueden considerar sensacionalistas (Acinas, Robles \& Peláez, 2015, p. 74). También se incluyen menciones a las notas de suicidios $(5,5 \%)$, aunque en menor proporción que la referida en otros estudios (Acosta, Milian \& Viña, 2012 Rátiva, Ruiz \& Medina, 2013, p.5; Muñoz \& Sánchez, 2013, p.16).

Se concluye que los diarios analizados realizan un tratamiento informativo sobre el suicidio consumado que podríamos calificar como dicotómico. Es 
decir, por un lado, los datos publicados son similares a los obtenidos en investigaciones realizadas desde el área hospitalaria, forense y de la psiquiatría, de modo que los medios operan como reflejo o espejo de la realidad. Sin embargo, al mismo tiempo, es un tratamiento alejado de las indicaciones de la OMS y que enfatiza aspectos sensacionalistas, dado que se ofrecen detalles relacionados con el mecanismo empleado y con la nota de suicidio, y que presentan el suicidio como la solución a un problema. Asimismo, se constata la ausencia de contenidos referidos a cuestiones como la prevención, caminos alternativos al suicidio, información sobre factores de riesgo, recursos comunitarios, líneas de ayuda y números de teléfono de grupos de apoyo, cuestiones recomendadas por la OMS. No obstante, futuras investigaciones debieran indagar si las indicaciones de la OMS (2017) son acogidas en la cobertura mediática de manera efectiva.

\section{Notas}

1 elpais.com;elmundo.es;abc.e;larazon.es

\section{Referencias Bibliográficas}

Acinas, M. P., Robles, J. I. \& Peláez, M. A (2015). Nota suicida y autopsia psicológica: Aspectos comportamentales asociados. Actas Españolas de Psiquiatría, 43 (3), pp. 69- 79.

Acosta A, Milian M, \& Viña H. (2012). Factores de riesgo del intento suicida en adolescentes con esta conducta. Revista Hospital. Psiquiátrico de la Habana. 9 (1), pp. 4-8.

Acosta, F. J., Rodríguez. C. J., \& Cejas, M. R. (2017). Noticias sobre suicidio en los medios de comunicación. Recomendaciones de la OMS. Revista Española de Salud Pública, 91, pp. 1- 17.

Arató, Demeter, Rihmer \& Somogy, (1988). Retrospective psychiatric assessment of 200 suicides in Budapest. Acta Psyhiatria Scandinavica, 77 (4), pp. 454-56. DOI:

10.1111/j.1600-0447.1988.tb05150.x

Ballús, C. (2006). Comentario editorial Suicidio y depresión. Humanitas Humanidades Médicas, 9, pp. 4- 5.

Bardin, L. (1986) Análisis de contenido. Madrid: Akal

Bardin, L. \& Suárez, C. (2002). El análisis de contenido (3a. ed.). Madrid: Akal.

Barraclough, B., Bunh, J., Nelson, B. \& Sainsbury, P. (1974). A hundred cases of suicide: clinical aspects. British Journal of Psychiatry, 125, pp. 355-373.

Berelson, B. (1952). Content analysis in communication researches. Michigan: Free Press.

Casado, M., Mata, P. \& Raya, A. (2012). Definición, datos estadísticos, sexo edad, Importancia de las cartas suicidas en la investigación forense. Cuadernos de Medicina Forense, 18, pp. 3- 4. DOli: http://dx.doi.org/10.4321/S1135-76062012000300004

Celis, A., Hernández, P., Gómez, Z., Orozco M.J. \& Rivas, M. (2004). Asfixia por sofocación y estrangulación en menores de 15 años. Gaceta Medica de México 140 (5)

Chávez, A, Macías, L. M. \& Luna, M.G. (2011). Notas suicidas mexicanas. Un análisis cualitativo. Pensamiento Psicológico 9 (17), pp. 33- 42. 
Dare, A. J., Andriessen, K. A., Nordentoft, M., Meier, M., Huisman, A. \& Pirkis, J. E. (2011). Media awards for responsible reporting of suicide: Experiences from Australia, Belgium and Denmark. International Journal of Mental Health Systems, 5, pp. 5-1 5. DOI: https://doi.org/10.1186/1752-4458-5-15

De la Gándara, J.J. (1995). El suicidio en niños y adolescentes. Monográfias de Psicología, 1 (7), pp. 34-40.

Dorpat, T. \& Ripley, H. (1960). A study of suicide in the Seattle area. Comprehensive Psychiatry, 1 (6), pp. 349- 359. DOI: https://doi.org/10.1016/S0010-440X(60)80058-2

Forti-Sampiero, L. \& Forti-Buratti, M.A (2012). Trastorno límite de la personalidad y conductas autolítica. Rev Esp Med Legal, 38, pp. 49- 54.

Garrido, F., Eleazar, A. \& Catalán, D. (2018). El uso por los periodistas de las recomendaciones de la OMS para la prevención del suicidio. el caso del periódico ABC. Revista Latina de Comunicación Social, 73, 810- 827. DOI: https://doi.org/10.4185/ RLCS-2018-1283

Gelder, M., Gath, D. \& Mayou, R. (1989). Suicide an deliberate selfharm. En: Gelder M., Gath, D., Mayou, R. (Eds.), Oxford Textbook of Psychiatry, pp. 478- 506. Oxford: Oxford University Press.

Gómez, E. L., Forti, M. A., Gutiérrez, B., Belmonte, A. \& Martín, C. (2016). Trastornos psiquiátricos en los casos de suicidio consumado en un área hospitalaria entre 2007-2010. Revista de Psiquiatría y Salud Mental, 9(1), pp. 31- 38. DOI: https://doi. org/10.1016/j.rpsm.2014.02.001.

González, A. (2015). Estrategias metodológicas para la investigación del usuario en los medios sociales: análisis de contenido, teoría fundamentada y análisis del discurso. El Profesional de la Información, 24, pp. 321- 328.

González, G. (2018). Hablemos del suicidio. Navarra: EUNSA

Gutiérrez, A. G., Contreras, C., \& Orozco, C. (2006). El suicidio, conceptos actuales. Salud Mental 29 (5), pp. 66- 74. Instituto Nacional de Psiquiatría Ramón de la Fuente Muñiz. Distrito Federal, México.

Hawton, K. \& Van Heeringer, K. (2009). “Suicide”. The Lancet, 373(1), pp. 1372- 1381. DOI: https://doi.org/10.1016/S0140-6736(09)60372-X

Herrera, R.; Ures, M. B. \& Martínez, J. J. (2015). El tratamiento del suicidio en la prensa española: ¿efecto werther o efecto papageno? Revista de la Asociación Española de Neuropsiquiatría, 35 (125), pp. 123- 134. DOI: https://doi.org/10.4321/S021157352015000100009

Holsti, 0. R. (1969). Content analysis for the social sciences and humanities. Reading, MA: Addison-Wesley.

Igartua, J. J. (2006). Métodos cuantitativos de investigación en comunicación (1a ed.). Barcelona: Bosch.

Instituto Nacional de Estadística INE (2017). Defunciones según la Causa de Muerte. www.ine.es/prensa/edcm_2017.pdf. 
Kripperndorf, K. (1980). Content analysis. An introduction to its methodology. Beverly Hills, CA: Sage.

Ladwig, K., Kunrath, S., Lukaschek, K. \& Baumert, J. (2012). The railway suicide death of a famous German football player: Impact on the subsequent frequency of railway suicide acts in Germany2. Journal of Affective Disorders, 136 (1-2), pp. 194- 198. DOI: https://doi.org/10.1016/j.jad.2011.09.044.

Landis, J. R., \& Koch, G. G. (1977). The measurement of observer agreement for categorical data. Biometerics, 33 (1), pp. 159-174.

Libro de Estilo de El País (2014). Santillana Ediciones. Madrid

Libro de Estilo del diario El Mundo (2002).

McCombs, M. (2006). Estableciendo la agenda: El impacto de los medios en la opinión pública y en el conocimiento. Barcelona: Paidós. Memoria del Fiscal General del Estado (2017).

Miguel, L., Lucena, F., Heredia, A., Rico, A. Marín, R., Blanco, M., Barrero, E., Soriano, M. T. \& Giménez, M. P. (2005). Estudio epidemiológico del suicidio en Sevilla en 2004. Cuadernos Medicina Forense, 11(39), pp. 43- 53.

Mínguez, L., García, I. \& De la Gándara, J. J. (2010). Suicidio, el último verso de un poeta. Norte de Salud Mental, 36, pp. 143- 152.

Motto, J. (1967). Suicide and suggestibility. American journal of psychiatry, 124, pp. 252 256.

Morcillo, L., Saíz, P., Bousoño, M. \& Bobes, J. (2000). Análisis epidemiológico y manejo de las conductas suicidas. En: Barcia Salorio, D. (Ed), Tratado de Psiquiatría, pp. 801- 819. Madrid: Arán Ediciones SA.

Müller, F. (2011). El “Efecto Werther”: gestión de la información del suicidio por la prensa española en el caso de Antonio Flores y su repercusión en los receptores. Cuadernos de gestión de Información, 1, pp. 65-71.

Muñoz, L. F. \& Sánchez, R. (2013). Caracterización de noticias sobre suicidio en medios impresos en Colombia. Revista Colombiana de Psiquiatría, 42(1), pp. 12- 18.

Navarro, N. (2017). El suicidio en jóvenes en España: Cifras y posibles causas. Análisis de los últimos datos disponibles. Clínica y Salud, 28(1), pp. 25-31. DOI: https://doi. org/10.1016/j.clysa.2016.11.002

Nock, M. K., Borges, G., Bromet, E. J., Cha, C. B., Kessler, R. C., Berelson, B. \& Lee, S. (2008). Suicide and suicidal behavior. Epidemiol Revista, 30, pp. 133- 154.

Olmo, A. \& García, D. (2015). Suicidio y libertad de información: entre la relevancia pública y la responsabilidad. Revista de Comunicación de la SEECI, 38. DOI: http://dx.doi. org/10.15198/seeci.2015.38.70-114.

Organización Mundial de la Salud. OMS (2000). Prevención del suicidio un instrumento para profesionales de los medios de comunicación. Ginebra. 
Organización Mundial de la Salud. OMS (2003). Informe mundial sobre la violencia y la salud. Ginebra

Phillips, D. P. (1974). The influence of suggestion on suicide: Substantive and theoretical implications of the Werther effect. American Sociological Review, 39 (3), 3pp. $40-$ 354. DOI: https://doi.org/10.2307/2094294.

Phillips, D. P, Lesnya K, Paight D. J. (1992). Suicide and media. IN: Maris RW, Berman AL, Maltsberger JI, eds. Assessment and prediction of suicide. New York, Guilford, pp. 499-519.

Piñuel Raigada, J. L. \& Gaitán Moya, J. A. (1995). Metodología general. Conocimiento científico e investigación en la comunicación social. Madrid: Síntesis.

Piñuel Raigada, J.L. (2002). Epistemología, metodología y técnicas del análisis de contenido. Estudios de Sociolingüística 3 (1), pp. 1- 42.

Pirkis, J. \& Blood, RW. (2001). Suicide and the media. part I: Reportage in nonfictional media. Crísis, 22 (4), pp. 146- 54.

Pirkis, J., Blood, R. W., Beautrais, A., Burgess, P. \& Skehan, J. (2006). Media guidelines on the reporting of suicide. Crisis: The Journal of Crisis Intervention and Suicide Prevention, 27 (2), pp. 82- 87

Rátiva, J. S., Ruíz, V. A. \& Medina, O. A. (2013). Análisis de las noticias sobre suicidio publicadas en un diario local del Quindío, Colombia, entre 2004 y 2011. Revista Cubana de Higiene y Epidemiologia, 51 (2), pp. 184- 191.

Rich, C.L., Ricketts, J. E. Fowler, R. C. \& Young, D. (1988). Some differences between men and women who commit suicide. The American Journal of Psychiatry. 145 (6), pp. 718-22. DOI: 10.1176/ajp.145.6.718

Robledo, M. M., Koutsourais, I., López, A., Serrano, M., Villareal, J \& Martín, D. (2015). Estudio médico legal del suicidio en España en el periodo 2000-2012. Derecho y Cambio Social, 12 (39), pp. 1-10

Romero JL, \& Gamero JJ. (2005). El suicidio consumado en el partido judicial de Huelva. Cuadernos de Medicina Forense, 11,pp.5-9.

Roy, A., Nielsen, D., Rylander, G., Sarchiapone, M. \& Segal, N. (1999). Genetics of suicide in depression. Journal of Clinical Psychiatry, 60 (2), pp. 12- 20.

Rubiano, H., Quintero, T. \& Bonilla, C. I. (2007). El suicidio: más que una noticia, un tema de responsabilidad social. Palabra Clave, 10 (2), pp. 93- 111.

Ruíz Pérez, I. \& Olry de Labry Lima, A. (2006). El suicidio en la España de hoy. Gaceta Sanitaria, 20 (1), pp. 25- 31. DOI: https://doi.org/10.1157/13086023.

Sadock, B. \& Sadock, V. A. (2008). Suicidio. En Kaplan, H. I. \& Sadock, B. J. (Eds.), Sinopsis de psiquiatría, 897-907. Barcelona: Wolters Kluwer Lippincott Williams y Wilkins. 
Sánchez, R., Orejarena, S. \& Guzmán Y. (2004). Características de los suicidas en Bogotá: 1985-2000. Revista de Salud Pública 6 (3), pp. 217-234.

Santurtún, M., Santurtún, A., Aguado, A. \& Zarrabeitic, M. T, (2016). Método empleado en las muertes por suicidio en España: envenenamiento y agentes violentos no tóxicos. Cuadernos de Medicina Forense, 22, 1-8.

Scherr, S. \& Steinleitner, A. (2015). Zwischen dem Werther- und Papageno-Effekt. Nervenarzt, 86, 557-565.

Sisask, M. \& Värnik, A. (2012). Media roles in suicide prevention: a systematic review. Int. J. Environ. Res. Public Health 9, 123-138.

Sudak, H. S. \& Sudak, D. M. (2005). The media and suicide. Academic Psychiatry, 29(5), 495-499.

Tejedor, C. \& Sarró, B. (2005). Conducta suicida. En: Soler, P. A, Gascón, J. (Coords), RTMIII. Recomendaciones terapéuticas en los trastornos mentales, 431-447. Barcelona: Grupo Ars XXI de Comunicación SL.

Villanueva, E. \& Hernández, A. F. (2004). Toxicocinética. En: Villanueva E, editor. Medicina Legal y Toxicología. 6. a ed. Barcelona: Masson, 708-26.

Wimmer, R. \& Dominick, J. (1996). La investigación científica de los medios de comunicación. Una introducción a sus métodos. Barcelona: Bosch.

World Health Organisation (2008). Department of Mental Health and Substance Abuse. Preventing Suicide: A Re- source for Media Professionals. Ginebra; 2008.

World Health Organization (WHO) (2017). Preventing suicide: a resource for media professionals, update 2017. Geneva. Retrieved from http://apps.who.int/iris/bitstream/10665/258814/1/WHO-MSD-MER-17.5-eng.pdf?ua=1.

\section{- Sobre la autoras}

María Teresa Santos es doctora en Ciencias de la Información UPV/EHU. Es Profesora Titular en la Facultad Ciencias Sociales y de la Comunicación (UPV/EHU). Autora de publicaciones sobre el tratamiento de diversos temas de salud en prensa, periodismo en redes sociales, comunicación y medios locales (prensa, radio, televisión), entre otras.

Idoia Camacho es doctora en Ciencias de la Información UPV/EHU. Es Vicedecana de Comunicación y Relaciones Externas de la Facultad de Ciencias Sociales y de la Comunicación (UPV/EHU). Autora de publicaciones sobre el tratamiento de diversos temas sanitarios en la prensa, periodismo en redes sociales y comunicación corporativa, entre otras.

- ¿Como citar?

Santos, M. T. \& Camacho, I. (2019). Suicidio consumado y representación de sus factores desencadenantes en la prensa generalista española en 2017. Comunicación y Medios, (40), 28-41. 